\title{
EchoGéo
}

$56 \mid 2021$

Les dynamiques spatiales contemporaines de la Russie

\section{Métropolisation, inégalités sociales et modes d'habiter : trajectoire d'une ville millionnaire russe vue par ses dačniki}

Le cas de Kazan

\section{Camille Robert-Boeuf}

\section{(2) OpenEdition}

Journals

\section{Édition électronique}

URL : https://journals.openedition.org/echogeo/21830

DOI : 10.4000/echogeo.21830

ISSN : 1963-1197

Éditeur

Pôle de recherche pour l'organisation et la diffusion de l'information géographique (CNRS UMR 8586)

\section{Référence électronique}

Camille Robert-Boeuf, « Métropolisation, inégalités sociales et modes d'habiter : trajectoire d'une ville millionnaire russe vue par ses dačniki », EchoGéo [En ligne], 56 | 2021, mis en ligne le 25 juin 2021, consulté le 03 août 2021. URL : http://journals.openedition.org/echogeo/21830 ; DOI : https://doi.org/ $10.4000 /$ echogeo. 21830

Ce document a été généré automatiquement le 3 août 2021.

EchoGéo est mis à disposition selon les termes de la licence Creative Commons Attribution - Pas d'Utilisation Commerciale - Pas de Modification 4.0 International (CC BY-NC-ND) 


\title{
Métropolisation, inégalités sociales et modes d'habiter : trajectoire d'une ville millionnaire russe vue par ses dačniki
}

Le cas de Kazan

\author{
Camille Robert-Boeuf
}

\section{Introduction}

1 Si la Russie est un pays où la population est majoritairement urbaine depuis plusieurs décennies, avec un taux de $74 \%$, resté stable depuis $2000^{1}$, l'essentiel du processus d'urbanisation remonte au lancement des premiers plans quinquennaux à partir des années trente (Eckert, 2012 ; Kolossov, 2007). Nombre de villes ont été créées à l'époque soviétique et étaient à vocation industrielle : plus de 600 villes ont été fondées après 1917 et un tiers des villes actuelles l'ont été après 1945 (Vendina, 2007). Aujourd'hui encore, l'urbanisation de la Russie est caractérisée par l'importance des très grandes villes en plein essor économique et par les villes moyennes, souvent mono-industrielles et en difficultés (Eckert, 2012 ; Kolossov, 2007 ; Radvanyi, 2010).

2 La littérature scientifique a analysé les brutales conséquences de la chute de l'URSS sur l'organisation des villes (Eckert, 2012 ; Kolossov, 2007 ; Radvanyi, 2010). La crise socioéconomique des années $1990^{2}$ a pu mettre en péril, dans la plupart des pays de l'Est, l'hégémonie urbaine en provoquant des phénomènes d'exode urbain (Nefedova, 2013, 2015), amenant certains chercheurs, notamment russes, à parler de phénomènes de ruralisation (Brodač et Šilkin, 2019; Kutovoj, 2013; Parvu, 2016). Le passage à une économie de marché et la privatisation des structures urbaines ont complexifié la fabrique de la ville en raison de la multiplicité des enjeux socio-économiques (Kolossov, 1993). Plus récemment, les villes russes sont confrontées à des objectifs d'amélioration des infrastructures et du cadre de vie (Solodkij et Gorev, 2013; Žel'kovič, 2016), la 
question environnementale occupant une place prépondérante, en particulier pour les villes industrielles et les villes monofonctionnelles (Dussault, 2010 ; Klyuev et al., 2018 ; Rochet, 2017). Parallèlement, l'étalement des grandes villes et la gentrification des centres urbains posent la question des inégalités socio-spatiales. Les villes millionnaires voient émerger à la fois un changement du profil des quartiers centraux, où s'opère un remplacement des populations pauvres par des catégories socio-professionnelles plus aisées, et une transformation de leurs périphéries (Badyina et Golubchikov, 2005; Golubchikov et Badyina, 2006 ; Gorbachevskaya, 2019). Ces évolutions urbaines révèlent de nouveaux rapports de forces, autour de la fabrique de la ville, entre acteurs publics et privés (Blanc et Emelianoff, 2007 ; Carton de Grammont, 2013 ; Dussault, 2010).

3 La plupart des recherches académiques se fondent sur les exemples moscovite et pétersbourgeois, même quand le propos se veut généralisable à la Russie (Eckert, 2012). Cela peut s'expliquer par la suprématie de Moscou dans le système de villes russes (qui représente $17,6 \%$ du PIB national en $2019^{3}$ ) et par une meilleure accessibilité aux données concernant ces deux régions. Néanmoins, cette hégémonie de Moscou et, dans une moindre mesure, de Saint-Pétersbourg tend à diminuer depuis les années 2010 avec l'apparition de nouvelles «villes millionnaires ", une grande partie d'entre elles étant des capitales régionales (Cottineau, 2012). Kazan, capitale du Tatarstan, en est l'un des exemples.

4 Le Tatarstan est une république autonome située au cœur de la région de la Volga. Comme son nom l'indique, elle est le territoire historique des Tatars, un peuple d'origine turcique et de culture musulmane installé dans la région depuis le $13^{\mathrm{e}}$ siècle (Hilgemann, 2007) et qui, d'après le dernier recensement ethnique de 2010, représente $53,2 \%$ de la population totale de la région. Le Tatarstan entretient un rapport particulier au pouvoir central, contestant le fédéralisme russe depuis la chute de l'URSS et défendant son indépendance sous la forme d'un nationalisme tatar plus ou moins revendiqué (Radvanyi, 2018 ; Raviot, 1993). Kazan, sa capitale, est un outil central dans la stratégie de construction de l'identité tatare (Graney, 2007), comme l'illustre la grande mosquée Qolsarif qui fait face à la cathédrale de l'Annonciation au sein du Kremlin. De fait, pour les élites économiques et politiques de la région, la ville est devenue l'image de marque du Tatarstan, rivalisant symboliquement avec Moscou depuis l'anniversaire de son millénaire en 2005 (Faller, 2002; Graney, 2007). Plus encore, depuis les années 2010, Kazan a acquis un véritable rayonnement international, en accueillant plusieurs évènements sportifs internationaux, et en engageant sa rénovation à partir de 2005. Paradoxalement, elle conserve, plus que d'autres villes millionnaires, des caractéristiques issues de son passé soviétique avec la forte présence de collectifs de jardins (plus communément appelés datchas) qui se retrouvent au centre des mutations urbaines de la capitale régionale. De fait, ces regroupements de parcelles individuelles, où les populations urbaines produisent des fruits et légumes pour leur approvisionnement alimentaire et pour le loisir, sont situés dans les espaces les plus touchés par l'urbanisation et la métropolisation alors même qu'ils continuent d'être essentiels pour les populations issues de la classe moyenne ou populaire. Ainsi, les datchas cristallisent les enjeux socio-géographiques et environnementaux intraurbains et agissent comme des révélateurs des inégalités socio-spatiales de la ville.

5 À travers l'exemple de Kazan et l'étude de ses collectifs de jardins, cet article entend interroger les nouveaux enjeux urbains des grandes villes russes et la manière dont les modes d'habiter des dačniki ${ }^{4}$ y répondent ou s'en font l'écho. La notion de mode 
d'habiter, définie comme la manière dont les individus résident, travaillent et circulent au sein d'un territoire (Mathieu, 2017 ; Stock, 2012), a été choisie ici, car elle permet d'aborder les effets de la ville sur les habitants d'une manière générale, et de mettre en lumière des formes de réappropriation informelle des espaces urbains de la part des citadins.

6 Cet article retracera dans un premier temps l'évolution urbaine récente de Kazan et de ses datchas pour s'intéresser, dans un deuxième temps, à son impact sur les populations des collectifs de jardins en termes d'inégalités socio-spatiales, avant de voir comment, en réponse à ces inégalités, les pratiques informelles développées par les jardiniers construisent de nouveaux modes d'habiter.

7 L'étude se fonde sur des enquêtes de terrains réalisées dans le cadre d'un mémoire de Master puis d'une thèse de doctorat en géographie intitulée Les jardins collectifs : entre urbanisation de la campagne et agrarisation de la ville. Mise en regard de l'île-de-France et de Kazan et soutenue en 2019. Ce travail de recherche a permis de rassembler, entre 2012 et 2019, des données principalement qualitatives sur le terrain russe. Ont été réalisés 50 entretiens semi-directifs et discussions informelles avec des habitants de Kazan (principalement des dačniki) ainsi que 30 entretiens semi-directifs avec des acteurs publics, des architectes et paysagistes, et des associations locales et régionales de jardinage et environnementales. À cela s'ajoutent 100 questionnaires réalisés auprès des dačniki et 10 suivis plus spécifiques de famille de jardiniers, étendus sur plusieurs mois ou années, à partir d'entretiens de type récits de vie et de temps d'observation à leur domicile et dans leur datcha. Plusieurs documents d'urbanisme et statistiques ont été analysés et les données cartographiques collectées ont ensuite été vérifiées sur le terrain. Enfin, la méthode de l'observation participante a été pratiquée au cours de séjours annuels de 2012 à 2019 à Kazan. L'ensemble de ces enquêtes ont permis une analyse des évolutions de la ville de Kazan sur une décennie.

\section{Une modernisation et une internationalisation à marche forcée}

Bien avant les années 2000, Kazan était une ville importante à l'échelle régionale et nationale grâce à sa position commerciale stratégique sur la Volga et à son université, fondée en 1804 (Mathieu, 2017; Stock, 2012). Son rôle économique s'accroît considérablement pendant la Seconde Guerre mondiale, lorsqu'une grande partie de l'industrie de la région de Moscou est délocalisée à Kazan, afin de la protéger de l'avancée allemande. La population de la ville augmente et son plan général est profondément transformé (Zakirova, 2015) : à l'est et au nord apparaissent plusieurs quartiers concentrant des îlots d'habitat collectif et de grandes usines du secteur de la pétrochimie et de l'aviation militaire. C'est à cette période que les collectifs de jardins se multiplient à Kazan; l'État central facilite largement la création des lopins en donnant du foncier aux entreprises d'Etat (principalement du secteur militaroindustriel) qui sont chargées de les redistribuer à leurs employés. Ces entreprises se développent à Kazan au cours de la deuxième moitié du $20^{\mathrm{e}}$ siècle et créent de nombreux collectifs, certains regroupant plusieurs centaines de parcelles.

Les datchas en Russie et à Kazan

Les collectifs de jardins ou datchas (en russe sadovye tovariŝestva) sont des 
associations qui regroupent des lopins individuels où les citadins pratiquent le jardinage. Ces jardins se sont développés dès 1917 à Saint-Pétersbourg et à Moscou et après la seconde guerre mondiale dans les autres grandes villes russes (Lovell, 2003 ; Traven, 2004). Elles ont un impact sur la majorité des citadins russes, puisque certaines études ont estimé que $54 \%$ à $67 \%$ d'entre eux possèdent ou ont accès à une datcha (Boukharaeva \& Marloie, 2015 ; Boukharaeva \& Marloie, 2011). À l'époque soviétique, le foncier est la propriété de l'État et les collectifs sont gérés par les entreprises d'État. En 1998, les datchas acquièrent le statut d'association et, depuis la loi dite « d'amnistie des datchas » votée en 2006, un processus de privatisation des parcelles est en cours, transformant les lopins en propriété privée et laissant la gestion collective des espaces communs (voies de communication, infrastructures collectives, lacs ou espaces verts situés à l'intérieur des collectifs, etc.) aux structures associatives. Kazan compte environ 236 collectifs de jardins (Boukharaeva et Marloie, 2011) qui sont localisés dans les dents creuses du centre urbain et en périphérie, le long des axes de communication ou sur les berges de la Volga et de son affluent, la Kazanka. D'après nos enquêtes, ils concentrent une population de classe moyenne ou pauvre (la moyenne des revenus des enquêtés est un peu plus basse que le revenu moyen de la région de Kazan), ce sont majoritairement des femmes, des retraités ou de petits employés avec une moyenne d'âge de 48 ans. Les parcelles sont entretenues régulièrement par les jardiniers qui y viennent plusieurs fois par semaine en période estivale. Les retraités, eux, y vivent, du printemps à l'automne et ne retournent que rarement à la ville pendant cette période.

Illustration 1 - Exemple de datcha traditionnelle en périphérie de Kazan

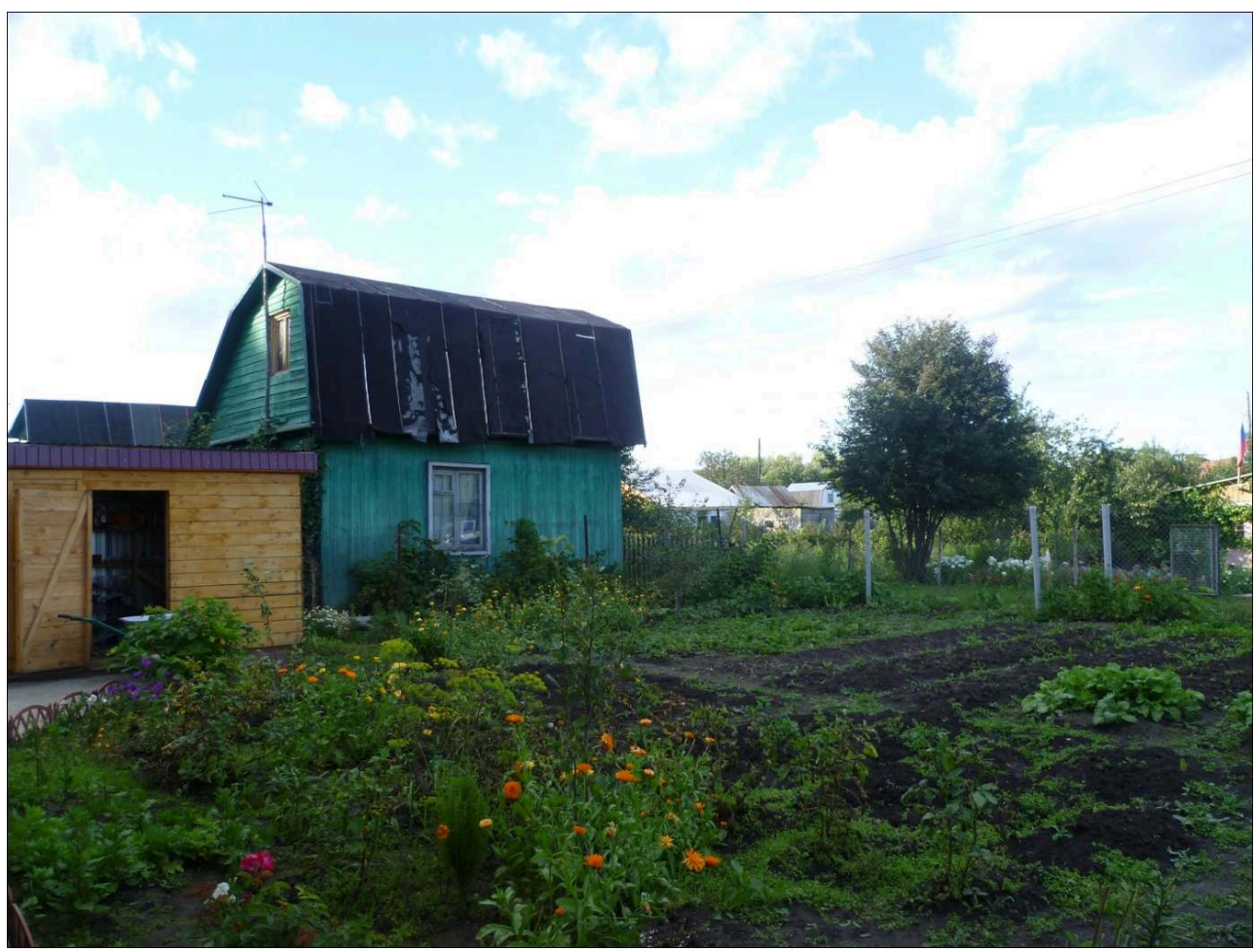

Auteur : C. Robert-Bœuf, 2015. 
La chute de l'URSS et la crise de 1998 fragilisent fortement les structures publiques gestionnaires de la ville et des datchas qui sont alors déstabilisées par la montée du crime organisé et par l'inflation. Les témoignages des habitants rencontrés sont nombreux : plusieurs d'entre-deux perdent leur travail et la majorité voient leur salaire diminuer drastiquement. Dans le même temps, les datchas deviennent centrales pour l'approvisionnement alimentaire, mais plusieurs collectifs de jardins disparaissent à la suite de la fermeture des entreprises d'Etat gestionnaires ${ }^{5}$. La situation s'est améliorée à partir des années 2000 et la création de la république autonome du Tatarstan, en 1992, a fini par bénéficier à Kazan qui accède à une position de pouvoir politique et administratif importante, en concentrant les ministères tatars.

À l'occasion du millénaire de la ville en 2005, son maire, Ilnour Raïssovitch Metchine, lance un vaste plan de rénovation et de modernisation du centre historique, rasant au passage plusieurs maisons traditionnelles en bois malgré la mobilisation de quelques associations locales, comme le racontent encore plusieurs enquêtés, membres d'associations ou architectes. C'est le début d'une nouvelle étape de transformation radicale qui vise à faire de Kazan une capitale moderne (Faller, 2002 ; Graney, 2007). Le processus s'accélère dans les années 2010 avec la tenue de plusieurs compétitions sportives internationales. La capitale tatare accueille les Universiades (compétition internationale universitaire multisports) en 2013, les compétitions internationales de natation en 2015 et plusieurs matchs de la Coupe du Monde de Football en 2018. En parallèle, les rénovations du centre historique mettent l'accent sur le patrimoine tatar de manière à réaffirmer la culture tatare (Graney, 2007), ainsi qu'à développer le tourisme intérieur. La rénovation du quartier historique tatar illustre parfaitement cette politique. Ce quartier a été réhabilité au milieu des années 2010 : les bâtiments ont tous été reconstruits et repeints avec des couleurs vives, produisant un effet de muséification du quartier. Des foires, marchés et manifestations culturelles y sont régulièrement organisés pour les touristes de passage (cf. illustration 2).

Illustration 2 - La rénovation du quartier tatar historique de Kazan sur les berges du lac Kaban

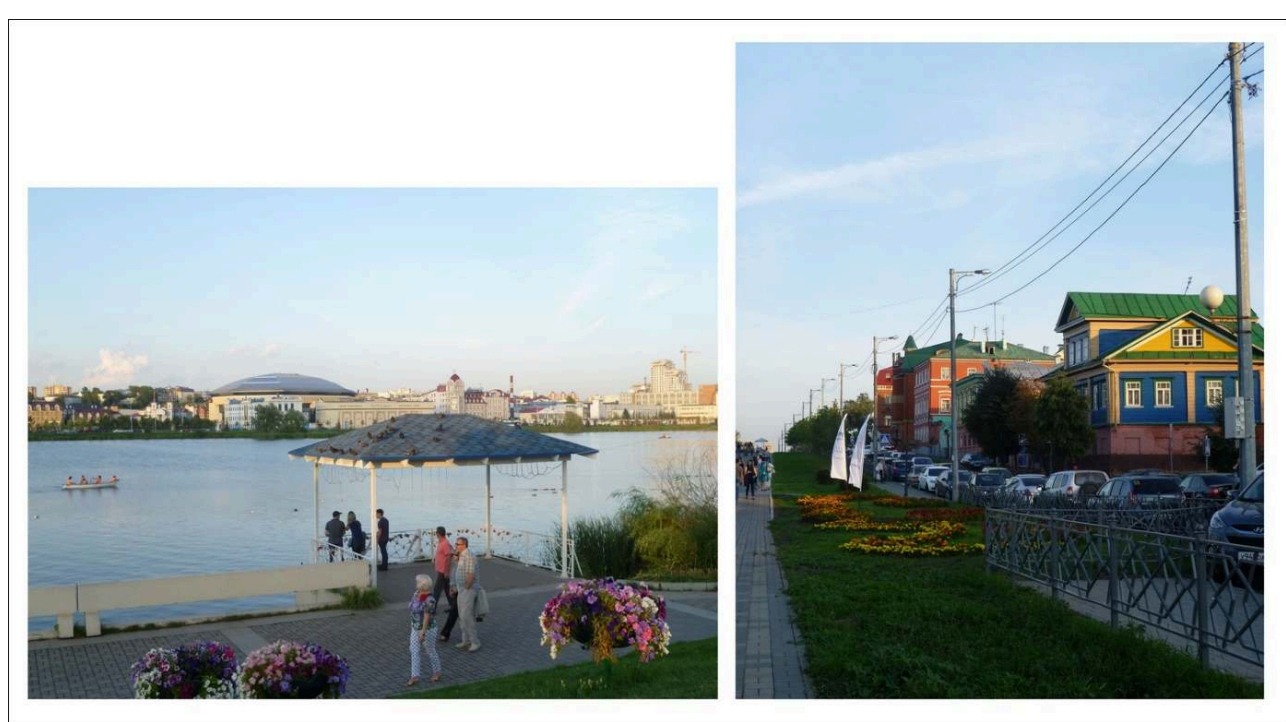

Ces deux photographies montrent la rénovation du quartier tatar historique.

À gauche : rénovation des berges du lac Kaban qui accueillent des marchés et évènements culturels. À droite : rénovation des maisons tatares traditionnelles en bois, repeintes avec des couleurs vives.

Auteur : C. Robert-Bœuf, 2015. 
11 Devenue capitale régionale touristique et culturelle, Kazan ne cesse également de s'internationaliser avec la volonté d'attirer des touristes étrangers, comme en atteste l'accord de coopération en matière de tourisme signé avec Paris en décembre 2016.

12 La volonté d'internationalisation de Kazan, de la part des autorités tatares, a entraîné l'amélioration et la modernisation des infrastructures (endettant par-là même durablement la municipalité) : remplacement de tous les vieux autobus par des neufs ; développement du réseau de transports avec de nouveaux tramways et extension de la ligne de métro; ou encore piétonnisation de certaines rues du centre historique. Mais cela a aussi beaucoup impacté les datchas et les pratiques des dačniki. La rénovation et la remise aux normes des marchés a, par exemple, contribué à la diminution du commerce informel des jardiniers les plus précaires qui y vendaient leurs légumes afin d'obtenir un complément de revenu. En outre, depuis 2010, les autorités locales s'attachent à réaménager les divers espaces verts de Kazan, sans toutefois prendre en compte les datchas. Ces dernières sont pourtant situées près des espaces réaménagés, sur les berges de la Kazanka ou près des lacs et poches de verdures (Nikitine et al., 2010 ; Nafisa et al., 2009), et sont considérées par la majorité des enquêtés comme des espaces naturels important du quotidien, ainsi qu'en témoigne Elena ${ }^{6}$, enseignante à la retraite et présidente d'un collectif de jardins :

«quand tu es dans le béton, tu as une sorte de sensation... tu ne peux sortir nulle part, et là-bas [à la datcha] tu y vas, et je ne sais pas l'expliquer, mais il y a une autre sensation. Une fois on a dormi là-bas sous la tente et quand je me suis réveillée, j'ai entendu tous les oiseaux, j'ai senti l'air, la végétation. Je ne voulais pas partir [...] Je vivrais bien toute l'année là-bas. Ce serait bien. »

13 Les collectifs de jardins du centre-ville ne sont alors pas intégrés aux politiques de rénovation de la ville et sont peu intégrés aux autres espaces ouverts.

L'essor économique de la capitale régionale a également impacté les datchas localisées en périphérie de la ville avec la périurbanisation des périphéries rurales. Kazan profite de son statut de capitale qui lui confère d'importantes fonctions économiques. Elle gère de manière indépendante un budget alimenté par l'exploitation des ressources pétrolières tatares, puisque le Tatarstan est le premier district pétrolifère de la Volga (Hilgemann, 2007). Kazan se trouve à la tête d'une région représentant $3 \%$ du PIB russe, ce qui la situe à la huitième position des 85 sujets que compte la Fédération de Russie. Cet essor économique a suscité un processus fulgurant d'urbanisation des périphéries de la ville, avec la multiplication des lotissements pavillonnaires, et l'élargissement de ses limites administratives, entre 2007 et 2010, le tissu urbain de Kazan empiétant désormais largement sur les rajons ${ }^{7}$ voisins au nord-est, à l'est et au sud (Ossipova et al., 2015). L'étalement de la ville est encouragé par les autorités municipales qui, selon le plan stratégique de développement socio-économique du Tatarstan 2030 et de l'avis de plusieurs enquêtés ayant participé à sa rédaction, souhaitent faire de Kazan une véritable métropole en construisant des villes satellites tout autour. La construction de la ville-satellite d'Innopolis, à l'ouest de Kazan, reflète l'intense périurbanisation des environs de la métropole. Cette ville située dans le rajon Verhneuslonskij, à environ $40 \mathrm{~km}$ du centre de Kazan (illustration 3), accueille plusieurs laboratoires de recherches et des entreprises de haute technologie, dans la perspective d'attirer des investisseurs et des chercheurs étrangers. 


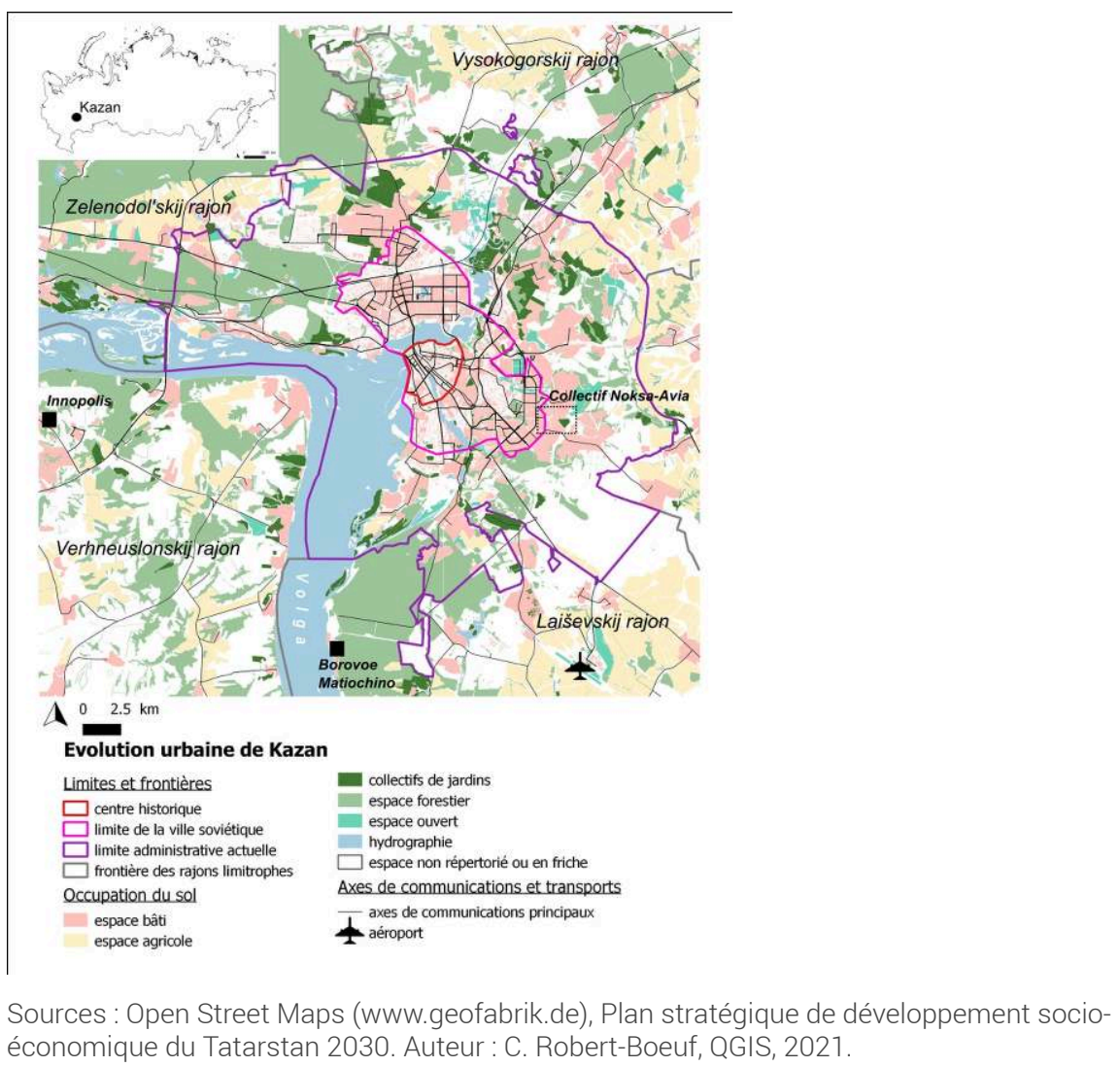

L'illustration 3 met en évidence les évolutions urbaines de Kazan et son étalement sur les périphéries rurales et sur les collectifs de jardins. On peut remarquer que de nombreux espaces sont encore en friches, au sud et à l'est de la capitale régionale. D'après les vérifications de terrain, ces friches (en blanc sur la carte) coïncident avec d'anciennes terres agricoles, utilisées comme réserves foncières, en attente de futurs projets de construction. De fait, si les terres agricoles sont normalement inconstructibles, toutes celles intégrées depuis 2010 à la métropole sont devenues constructibles, ce qui a eu pour conséquence d'entraîner d'importants mouvements de spéculation foncière.

Une partie importante de ces transformations urbaines se produisent dans les espaces investis historiquement par les datchas : dans les dents creuses de la ville, autour du centre urbain qui se densifie; dans les périphéries de la ville où de nouveaux quartiers sont construits. Ainsi, certains collectifs de jardins disparaissent ou sont grignotés petit à petit pour se transformer en quartiers pavillonnaires, essentiellement lorsqu'ils sont situés près du centre (Auteur, 2014). Dans ce contexte, les datchas sont les révélateurs privilégiés des fragilités et inégalités socio-spatiales de Kazan.

\section{Fragilités, inégalités socio-spatiales et contestations grandissantes}

17 La crise économique des années 1990 a suscité une réflexion sur les inégalités territoriales qui affectent encore aujourd'hui la société russe (Radvanyi et Laruelle, 
2016), et soulèvent la question de la pauvreté (Prokofieva, 2012 ; Prokofieva et al., 2003). Ces analyses s'appliquent aux inégalités entre les différentes régions russes ou entre les grandes et petites villes, et s'attachent moins à une analyse intra-urbaine des inégalités. Or, le cas de Kazan et de ses datchas révèle des phénomènes nouveaux de ségrégation socio-spatiale, les villes soviétiques ayant été jusque-là peu marquées par ce phénomène (Kolossov, 1993), et une aggravation des fragilités intra-urbaines avec le processus de métropolisation.

Le Tatarstan est une région relativement riche par rapport aux républiques voisines (Hilgemann, 2007). En 2015, le salaire moyen de la région de Kazan est de 460 euros (contre 440 euros pour la Russie), néanmoins, il faut noter qu'au Tatarstan 49,5\% de la population gagne moins de 391 euros par mois selon les statistiques officielles, ce qui montre une inégalité de revenus à l'échelle régionale. Ces chiffres sont confortés par nos enquêtes de terrain, car les dačniki rencontrés, qui appartiennent majoritairement à la classe moyenne et aux classes populaires, ont souvent un salaire encore inférieur dont une partie n'est pas déclarée, ce qui les rend très vulnérables aux réguliers phénomènes d'inflation. Les datchas étant localisées dans les espaces où l'urbanisation et les quartiers pavillonnaires se développent le plus, ces espaces jardinés sont révélateurs des évolutions inégalitaires de la ville.

Dans le centre-ville, la rénovation des bâtiments et la construction de nouveaux quartiers ont entraîné la création de plusieurs quartiers d'habitats collectifs pour des catégories sociales aisées. Cela a introduit une distinction spatiale entre les quartiers riches situés au centre et sur les berges de la Kazanka et les autres quartiers plus anciens, tout en provoquant une hausse globale des prix des logements à Kazan (Failovič et Igorevna, 2017). En périphérie, ce phénomène de ségrégation spatiale (Brun et Rhein, 1994), a introduit des formes de mises à l'écart de certains groupes sociaux, créant des effets de gentrification autour et à l'intérieur des collectifs de jardins (Brun et Rhein, 1994).

Autour des datchas s'implantent des lotissements pavillonnaires de luxe, véritables gated communities, notamment lorsque les jardins sont reliés à la ville et proches des berges de la Volga (illustration 4). La construction de ce type de lotissements pavillonnaires est difficilement quantifiable (du fait, parfois, de leur illégalisme), mais aussi parce que les données statistiques et foncières sont bien souvent inaccessibles. Cependant, l'étude du rajon Laiševskij, au sud de Kazan, est particulièrement éclairante pour comprendre le phénomène, car il réunit les critères de proximité du fleuve et de l'accès facilité au centre-ville tout en possédant historiquement une forte concentration de collectifs de jardins et de bases de loisirs (baza otdyha en russe), ce qui provoque des conflits d'usage entre collectifs de jardins et nouveaux quartiers. Ainsi, ces derniers se sont surtout installés sur les berges boisées de la Volga (pourtant officiellement inconstructibles), compliquant l'accès au fleuve aux collectifs de jardins situés plus en retrait. 
Illustration 4 - Exemple d'une gated community dans le rajon Laiševskij

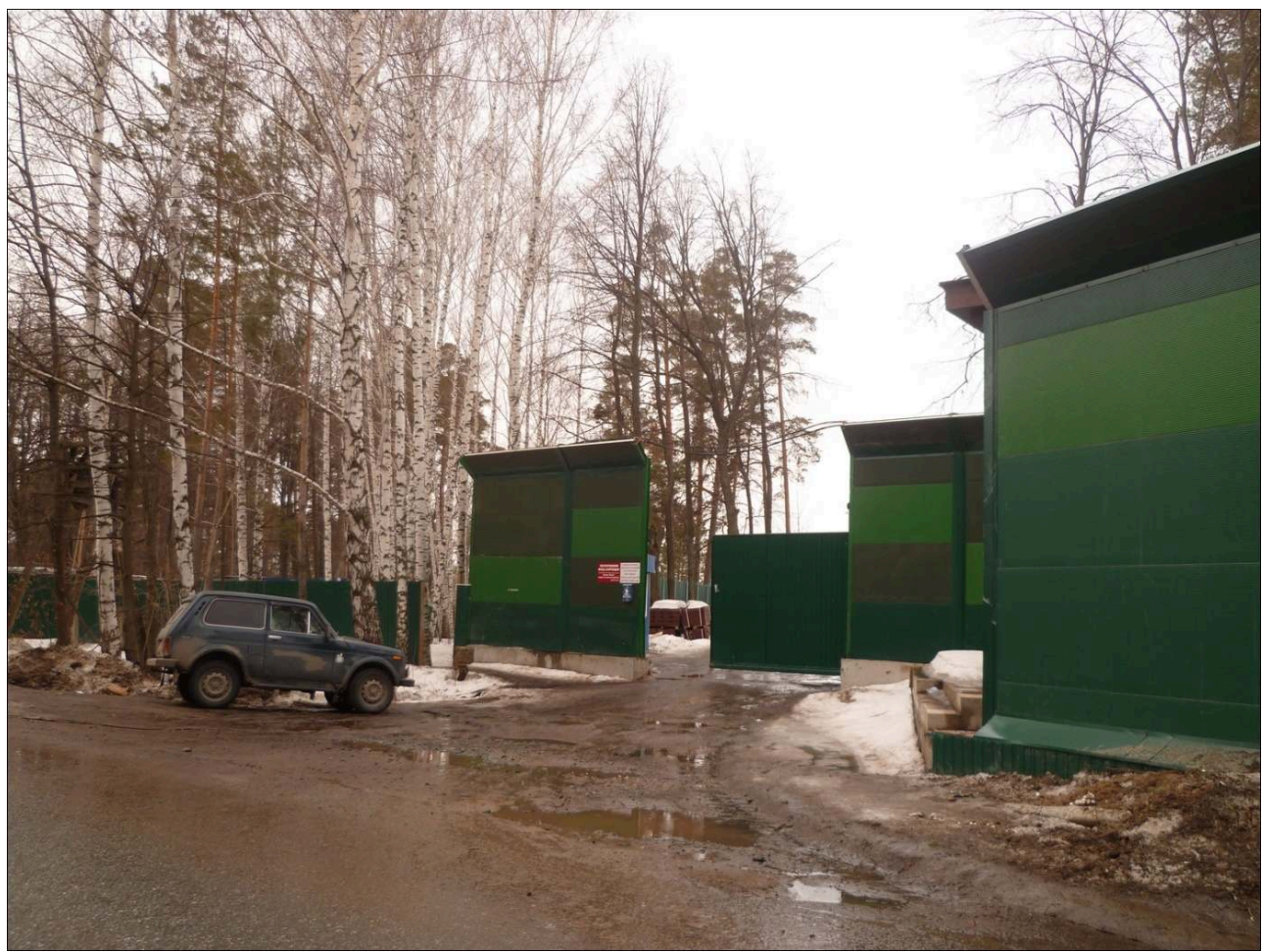

Cette photographie montre un quartier fermé du sud de Kazan où l'accès est strictement contrôlé et où seules les personnes autorisées peuvent pénétrer. Ces quartiers sont caractérisés par de hautes clôtures qui coupent le regard et privatisent de larges parties du périurbain de la capitale tatare.

Auteur : C. Robert-Boeuf, 2013.

Ces phénomènes de ségrégation spatiale font apparaître des formes d'inégalité sociospatiale et de distinctions spatiales entre d'une part des collectifs de jardins, héritiers de l'époque soviétique et concentrant essentiellement des populations pauvres, et d'autre part des lotissements pavillonnaires fermés accueillant des populations aisées ; comme le montre l'exemple du collectif de jardins Noksa-Avia. En 2013, à la suite de la vente illégale du foncier de ses voies de communication à un promoteur immobilier, ce collectif s'est trouvé entouré par plusieurs lotissements pavillonnaires. Ces derniers ont alors bloqué ses voies d'accès, ce qui a augmenté les risques d'incendie (puisque les parcelles n'étaient plus accessibles par les pompiers) et a rendu la desserte des parcelles très difficile (illustration 5). Il a fallu que la présidente du collectif de jardins intente un procès au promoteur immobilier pour pouvoir faire inscrire les voies d'accès du collectif au cadastre; l'enquête a ensuite montré que le foncier avait été vendu illégalement par un employé de l'organisme du cadastre. 


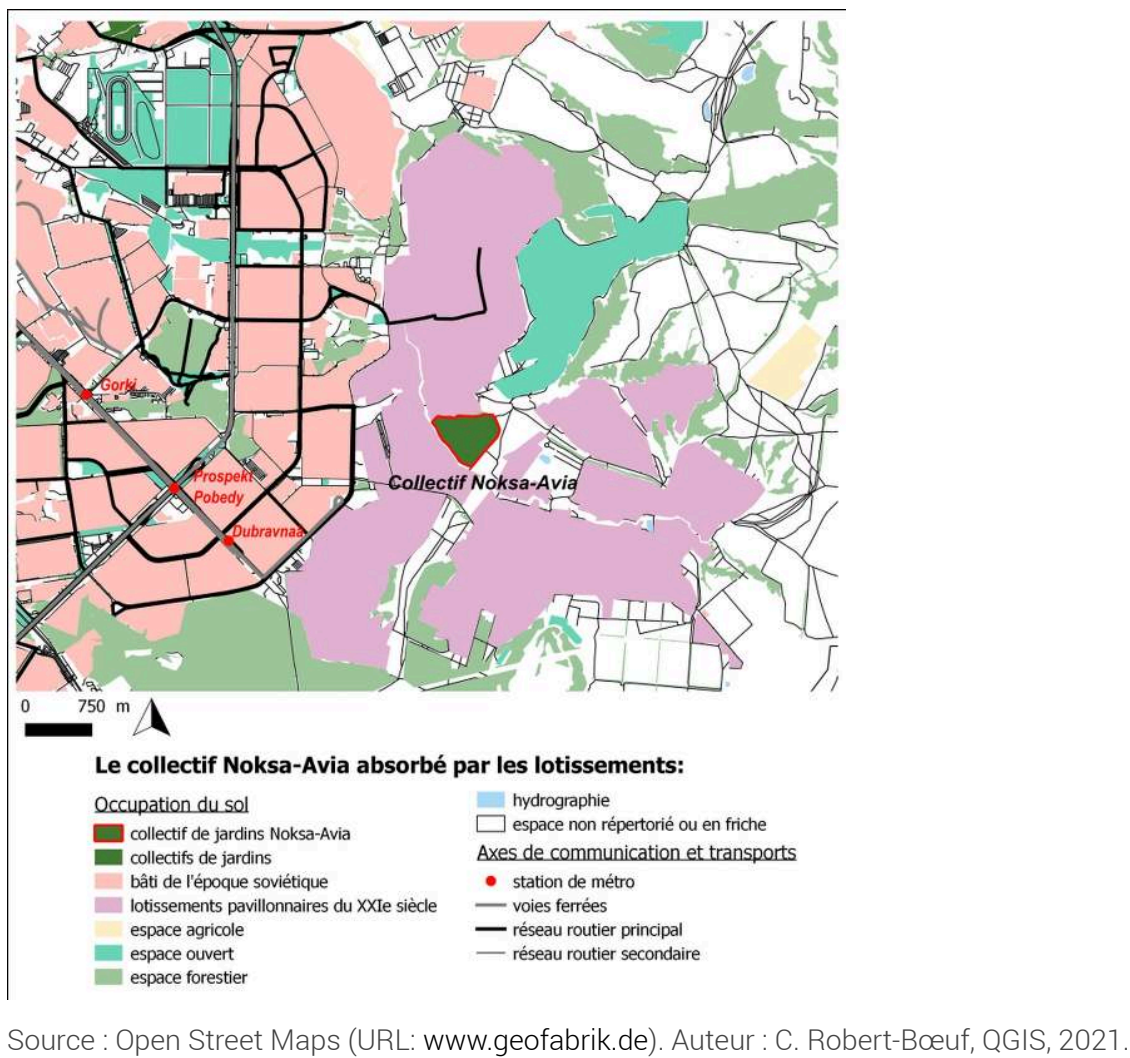

Cette ségrégation spatiale va de pair avec des processus de densification du bâti et d'urbanisation des périphéries rurales de Kazan. La création des lotissements pavillonnaires implique la rénovation des infrastructures, souvent de mauvaise qualité dans le périurbain et dans les communes rurales proches de Kazan, ce qui provoque l'augmentation des prix du foncier. En conséquence, les collectifs de jardins proches du front urbain, bien reliés au centre-ville et les plus proches de ces lotissements sont petit à petit absorbés par ceux-ci. Cette absorption peut se faire de deux manières : soit des acheteurs individuels achètent des parcelles et y construisent des pavillons ; soit les propriétaires des lopins eux-mêmes (ceux qui en ont les moyens) transforment leur parcelle et leur potager en résidence principale. De fait, des phénomènes d'embourgeoisement ou même de gentrification apparaissent au sein des jardins et les maisonnettes en bois traditionnelles sont remplacées par des cottages. C'est le cas près de Borovoe Matiochino, où se trouve aussi un ancien sanatorium qui attirait les populations aisées (illsutration 6), ce qui provoque le regroupement de populations aisées et l'exclusion des populations les plus pauvres8. 
Illustration 6 - Évolution de Borovoe Matiochino, commune du rajon Laiševskij

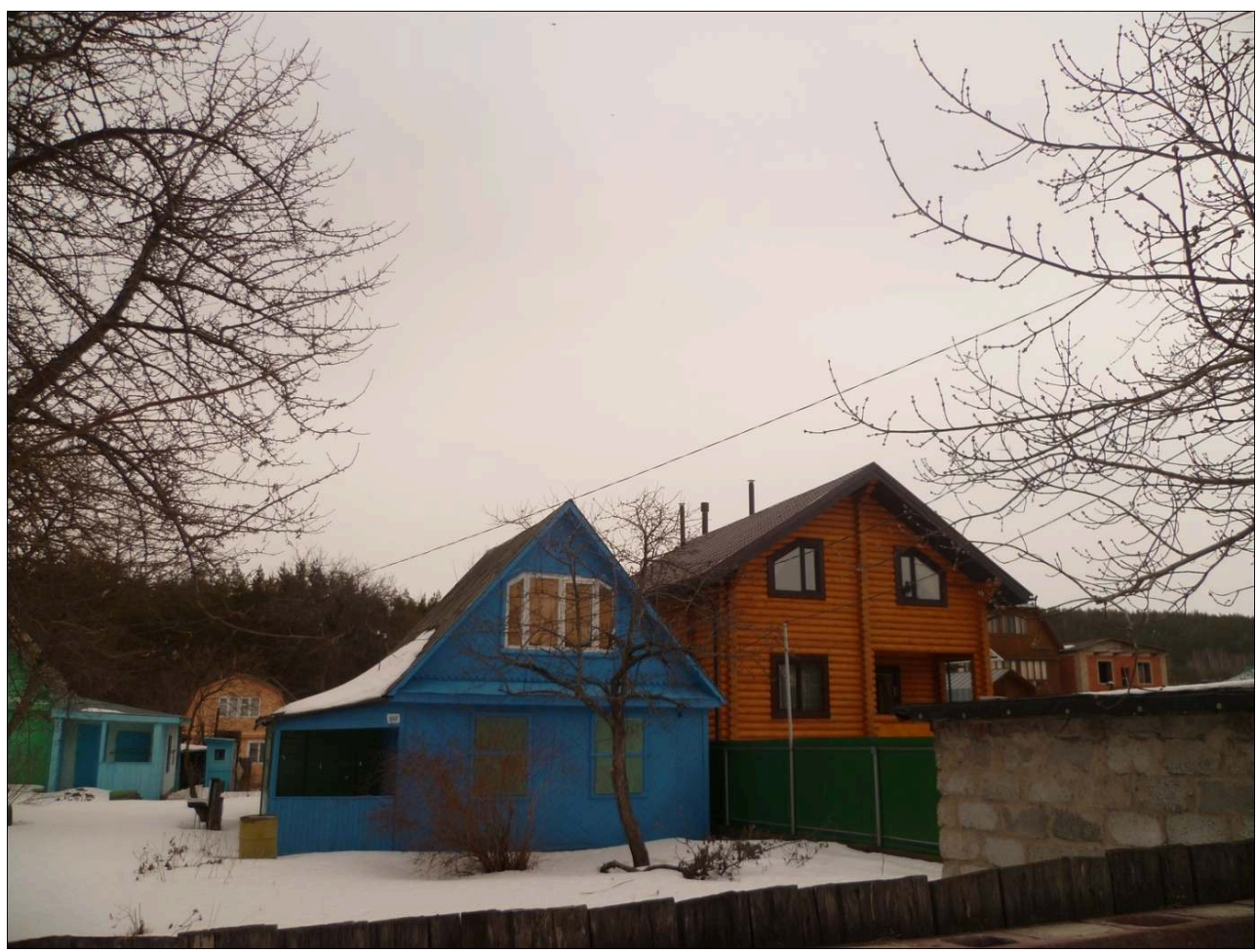

Cette photographie illustre l'urbanisation interne du collectif de jardins de Borovoe Matiochino dont les parcelles sont revendues à des populations aisées qui transforment les datchas traditionnelles en cottages. On y voit, à gauche, une datcha traditionnelle (petite, en bois et de couleur vive) et à droite un exemple de cottage moderne (de taille plus imposante, construit en dur).

Auteur : C. Robert-Boeuf, 2013.

L'exemple de l'illustration 6 atteste de l'apparition d'effets de gentrification, c'est-àdire de remplacement de populations pauvres par des populations plus aisées, comme cela a pu être décrit pour le cas moscovite (Badyina et Golubchikov, 2005 ; Golubchikov et Badyina, 2006 ; Gorbachevskaya, 2019). Cependant, il convient de nuancer le propos, car la gentrification ne concerne pour le moment que des quartiers très spécifiques de Kazan (Borovoe Matiochino étant autant emblématique qu'exceptionnel) et relève surtout de l'embourgeoisement, tandis que la majorité des mutations urbaines se caractérise plutôt par la formation de quartiers aisés séparés des quartiers plus populaires. Selon nos enquêtes, cette gentrification relève d'un processus complexe qui est étroitement lié au contexte local, comme cela a pu être analysé en géographie dans d'autres pays (Chabrol et al., 2016). À Kazan, cette gentrification se retrouve essentiellement dans les collectifs de jardins situés le long des principaux axes de communication menant au centre-ville et sur le front urbain, les datchas plus isolées étant moins touchées et préservant leurs lopins potagers.

Le développement des quartiers périphériques de Kazan se fait selon des impératifs économiques et spéculatifs qui ne correspondent pas aux pratiques et besoins réels des urbains, contribuant à un essor désordonné et finalement fragile de cette partie de la métropole. L'ambition de certains projets se heurte à l'instabilité économique de la région, ce qui affecte le territoire périurbain de la métropole. Certains chantiers, lancés en grande pompe, comme celui de la smart-city, n'ont, selon nos enquêtes, toujours pas abouti et quelques projets qui ont vu le jour sont quasi-inoccupés faute d'investisseurs 
ou d'acquéreurs, comme le Centre d'exposition international Kazan Expo, construit près de l'aéroport et ouvert en 2018, qui reste vide la majorité du temps, selon plusieurs urbanistes et géographes rencontrés.

Le même phénomène s'observe par la présence de lotissements pavillonnaires construits ex nihilo et en grand nombre, à l'ouest ou au sud de la capitale tatare, et qui sont en attente de propriétaires, les promoteurs ne parvenant pas à les vendre. Selon nos entretiens avec plusieurs habitants, architectes et urbanistes, cela s'expliquerait par le faible nombre de populations véritablement aisées, ainsi que par la relative précarité de la classe moyenne qui n'a pas les moyens d'acheter de pavillons suffisamment proches de la ville et qui préfère conserver un appartement en centreville et rénover la datcha pour en faire une véritable résidence secondaire. En effet, selon le questionnaire distribué auprès des dačniki, 96 sur 100 déclarent vouloir conserver leur datcha et 83 ne souhaitent pas avoir une autre parcelle, même avec un cottage, ou disent ne pas en avoir les moyens. Ces jardiniers sont représentatifs d'une partie de la population qui, malgré l'essor économique important depuis les années 2000 de la région, reste en situation précaire en raison de l'inflation qui augmente le coût de la vie. Kazan semble donc en proie à des logiques spéculatives de la part de promoteurs immobiliers qui ont entrainé l'explosion des quartiers pavillonnaires et qui ne répondent pas à la demande de la population ${ }^{9}$. Ces dynamiques font écho à celles, très semblables, analysées à Moscou et à Kiev, au début des années 2000 (Makhrova, 2014 ; Nefedova et Savtchouk, 2014 ; Traven, 2004), ce qui indique un certain décalage du cas de Kazan par rapport aux évolutions foncières de Moscou, il y a quelques années. Cette fragilité toujours visible met donc à mal l'image d'une capitale régionale au processus urbain et métropolitain triomphant.

De plus, la transformation de la ville et de ses quartiers périphériques ne prend pas en compte les problèmes environnementaux qui en résultent et la réaction des citadins qui s'exprime face à certains aménagements. Les grands travaux issus des compétitions sportives entre 2013 et 2018 ont provoqué la contestation des populations locales et des associations écologiques qui ont été peu consultées et peu intégrées dans les processus de décision quant aux aménagements urbains (Ermolaeva, 2014); mais aussi de la part de la communauté scientifique qui a notamment étudié l'impact socio-économique et environnemental des Universiades (Bagautdinova et al., 2015). Ces études établissent que les citadins ont été peu consultés à l'occasion des aménagements récents de la ville et que ces derniers ont eu un impact environnemental important. Si les effets de la croissance économique ont pu être positifs, la transformation de la métropole et l'accueil de grands évènements sportifs ont causé une hausse de la pollution atmosphérique et une dégradation des espaces naturels et des lacs (Bagautdinova et al., 2015). Comme dénoncé dans le cas d'autres villes dans le monde (Collinet et Schut, 2020; Junod, 2007), les infrastructures dédiées aux compétitions sportives internationales ont également eu un coût conséquent et sont maintenant sousexploitées, alors que les infrastructures quotidiennes et les quartiers d'habitations voisins souffrent d'un manque d'investissements.

Plus largement, l'étalement de Kazan entraîne des dégâts environnementaux, ce qui provoque également des tensions entre Kazan et les communes voisines, la gestion des déchets des jardins collectifs en étant un bon exemple. Ces dernières années, les datchas sont critiquées par les populations des communes périurbaines où elles sont implantées, car la gestion de leurs déchets est souvent mal organisée et les déchets de 
dačniki polluent l'environnement sans que les collectivités locales ne disposent du budget suffisant pour y remédier. Ces enjeux environnementaux de la capitale tatare mettent en lumière une fabrique de la ville qui s'opère sans la participation de ses habitants et qui introduit, dans le même temps, des inégalités entre les populations pauvres et celles plus aisées, entre les populations du centre-ville et celles qui habitent en périphérie.

\section{Pratiques informelles et réappropriation par les citadins-dačniki : un autre mode d'habiter la ville}

Face à ces mutations urbaines, aux fragilités et inégalités qui en découlent, les citadins, et en premier lieu les dačniki, se saisissent de certains espaces urbains afin de développer des modes d'habiter qui répondent mieux à leurs besoins. Le concept de mode d'habiter, qui a été défini par N. Mathieu dans le cadre de ses travaux sur les relations ville-campagne (Mathieu, 2017), se situe au croisement de deux notions, l'une géographique, le "genre de vie », l'autre sociologique, le «mode de vie " (Mathieu, 2007), ce qui autorise une analyse plus globalisante des pratiques citadines. Dans le cas de la Russie, cela permet de prendre en compte des comportements individuels ou collectifs souvent informels qui se structurent à une échelle micro-locale et d'analyser leur impact à l'échelle métropolitaine alors même qu'ils sont peu considérés par les autorités publiques et généralement étudiés à travers le seul prisme environnemental (Blanc et Emelianoff, 2007).

Nous avons vu que Kazan s'était beaucoup modernisé, à l'exception des quartiers d'habitat collectif de l'époque soviétique où vivent l'ensemble des dačniki rencontrés (sauf une exception), et où se concentre une majorité de la population urbaine. Ces logements sont peu rénovés, pourtant, ils peuvent être remobilisés par les jardiniers eux-mêmes. Au cours de nos entretiens avec les dačniki, nous avons rencontré plusieurs femmes qui s'investissent dans le quartier de leur résidence principale, ce que des observations dans divers quartiers de Kazan ont confirmé. Nous avons ainsi réalisé un entretien avec Nela, sexagénaire à la retraite qui habite dans le quartier NovoSavonovski, au nord de la Kazanka. Au cours de la journée passée en sa compagnie, elle raconte comment elle a transformé la cour intérieure de son immeuble, autrefois envahie par un parking illégal, en un espace vert. Fermant petit à petit les places de parking en y cultivant des fleurs, elle a sensibilisé ses voisins et a réussi à interpeler, devant les caméras de journalistes, les autorités locales, qui ont fini par légitimer cet espace vert en y plantant un grand sapin qu'elle nous montre avec fierté. Nela a donc su, avec quelques voisines, intervenir d'abord de manière informelle pour améliorer son cadre de vie, puis elle est parvenue à officialiser son action grâce à l'envoi de courriers et à la mobilisation des journaux locaux. Cet exemple fait écho à l'article de N. Blanc et C. Emelianoff (2007) sur les mobilisations environnementales à Tver et Nijni Novgorod qui décrivent des initiatives semblables . Nos enquêtes confirment que cette réappropriation, plus ou moins officielle, est bien présente dans les grandes villes millionnaires et s'y maintient, alors même que la municipalité s'empare de ces questions en rénovant depuis 2015 l'ensemble des parcs de la ville. La confirmation de ce type de pratiques et d'interventions à l'échelle micro-locale indique que ce qui est recherché, ce n'est pas seulement l'implantation d'espaces verts de qualité, mais audelà la réappropriation de l'initiative d'aménagement de l'espace résidentiel urbain, 
comme le soulignait l'article de N. Blanc et C. Emilianoff (2007). À Kazan, cela semble particulièrement vrai aujourd'hui pour les immeubles soviétiques dont les infrastructures n'ont pas été rénovées. Ces formes de réappropriation des espaces collectifs vont au-delà d'une simple défense de l'environnement et du cadre de vie urbain, puisque que ces actions construisent des modes d'habiter qui permettent de se nourrir, d'habiter et de faire communauté en ville, de manière différente.

Notre travail de terrain a montré que les datchas sont encore majoritairement agricoles et que les citadins les cultivent afin de se nourrir autrement (Robert-Boeuf, 2017). Les datchas fournissent ainsi une alimentation locale, de meilleure qualité, variée selon les saisons à une partie non négligeable de la population urbaine, puisqu'une majorité d'entre elle a accès à une datcha (Boukharaeva et Marloie, 2011; Robert-Bœuf, 2017, 2019). Ces pratiques s'intègrent dans des stratégies plus larges d'approvisionnement alimentaire des familles urbaines qui mobilisent des réseaux d'interconnaissance à l'échelle des campagnes périurbaines pour se procurer des produits laitiers et carnés de meilleure qualité. Ces circuits très informels ne permettent pas un approvisionnement régulier, mais donnent accès à des produits de meilleure qualité, pour des populations appartenant aux classes moyennes ou pauvres. C'est ainsi que les enquêtés peuvent commander à l'avance de la viande qu'ils congèlent de manière à en disposer tout au long de l'année. À l'échelle d'une métropole de la taille de Kazan, bien approvisionnée par les circuits alimentaires classiques, il est intéressant de voir que nombre d'habitants développent des stratégies individuelles afin d'accéder à une nourriture de qualité, dans un contexte où la méfiance des circuits traditionnels est héritée de l'époque soviétique et où les magasins bio sont peu présents, chers et sans labellisation officielle en Russie.

31 Ces stratégies d'adaptation se retrouvent également dans la manière dont les urbains utilisent leur datcha afin de profiter d'une bi-résidentialité au quotidien. Cette birésidentialité, entre l'appartement et le lopin individuel et les mobilités qui y sont associées leur permettent de construire un espace de vie plus conforme à leurs besoins et aspirations. Les citadins sont alors transformés en homo mobilis, décrits par le géographe A.Treïvish, et effectuent des déplacements réguliers entre centre et périphérie de la métropole (Droujinin et al., 2012). Pour les populations urbaines les plus pauvres, c'est un moyen d'acquérir un pavillon individuel, souvent considéré comme l'habitat idéal dans les entretiens, tout en conservant un appartement proche du centre-ville et du lieu de travail. C'est, pour beaucoup, une « réponse à la crise et à la précarité par la mobilité et la flexibilité » (Mathieu, 2017). Si cette bi-résidentialité pouvait être considérée comme subie à l'époque soviétique, puisque les dačniki ne choisissaient pas la localisation de leur parcelle, elle est de plus en plus choisie, car ils peuvent soit vendre leur appartement du centre-ville pour acquérir un cottage en périphérie (ce qui a été le cas pour quelques enquêtés) ou acheter une parcelle de jardins ailleurs. Cependant, nous observons que les urbains ont tendance à maintenir cette bi-résidentialité et à l'entretenir, car elle favorise une meilleure adaptabilité entre centre et périphérie et offre un accès au foncier relativement peu cher.

32 Ces pratiques informelles d'adaptation à la ville vont de pair avec la formation, à l'échelle des quartiers d'habitation et des collectifs de jardins, de communautés d'interconnaissance et d'entraide. Comme cela a pu être conceptualisé par S. Carton de Grammontb $(2013,2018)$, les urbains ont tendance à faire appel à des «régimes de voisinage » qui renforcent des réseaux d'entraide à l'échelle micro-locale et à l'échelle 
métropolitaine. Les stratégies de réappropriation des cours intérieures, d'approvisionnement alimentaire et de bi-résidentialité sont entretenues par des communautés qui combinent différentes sociabilités : les sociabilités de voisinage (à l'échelle d'un quartier), les sociabilités familiales (entre différents quartiers et entre ville et campagne) et les sociabilités jardinières (au sein des collectifs de jardins).

Le croisement de ces stratégies et de ces diverses communautés donne naissance à des modes d'habiter particuliers qui permettent aux populations urbaines de revendiquer leur place au sein d'une ville qui se fabrique sans eux, créant un véritable droit à la ville (Lefebvre, 1968), à ses espaces résidentiels et ses espaces verts. Par les pratiques agricoles qu'elles entretiennent, les datchas participent aussi d'un droit d'accès à la terre nourricière. Ainsi, par le développement de modes d'habiter propres, les populations des classes moyennes et pauvres accèdent à un «droit à la vie urbaine, transformée et renouvelée » (Lefebvre, 1968, p. 132). Ces modes d'habiter répondent à «des besoins de lieux qualifiés, lieux de simultanéité et de rencontres, lieux où l'échange ne passerait pas par la valeur d'échange, le commerce et le profit » (Lefebvre, 1968, p. 116). Dans le cas de Kazan, ce droit à la ville a la spécificité d'être complété par un droit à la nature (Boukharaeva et Marloie, 2011) et à la terre qui sont entretenus en partie par la présence des datchas et par la manière dont les urbains réinvestissent leurs espaces verts de proximité. De fait, le travail de la terre et les pratiques jardinières s'avèrent aussi importants que la création ou la préservation d'espaces de nature, comme en témoigne Olga, jardinière sexagénaire et ancienne journaliste :

«Je jardine parce que comme ça je sais comment ça pousse, si j'ai rajouté des engrais ou pas, s'il y a des engrais chimiques ou pas. [...] Je sais que toutes mes productions sont écologiques. Ce qui me plait le plus c'est voir les fleurs éclosent, c'est-à-dire voir le résultat. Quand les fleurs fleurissent et quand mes légumes donnent bien. »

Ce besoin de constater l'impact de son action dans le jardin et son "résultat " se retrouve largement chez les dačniki rencontrés, puisque selon nos questionnaires, 65 jardiniers sur 100 estiment que la datcha est avant tout un lieu de production et vivrier. Ainsi, nos enquêtes soulignent l'attachement des urbains à un savoir-faire agricole et à des pratiques au contact de la pleine terre qui font la distinction entre avoir accès à un espace vert et entretenir cet espace au quotidien.

\section{Conclusion}

L'évolution urbaine de Kazan étudiée à travers le prisme des collectifs de jardins et de leurs enjeux spatiaux, sociaux et environnementaux met en exergue les inégalités socio-spatiales auxquelles sont confrontées les villes millionnaires russes. Néanmoins, le cas de Kazan demeure particulier, car ses mutations urbaines sont particulièrement rapides alors que les datchas jouent encore un fort rôle alimentaire pour les habitants de la capitale tatare ; contrairement à Moscou ou Saint-Pétersbourg où les collectifs de jardins se sont en grande partie transformés en lotissements et résidences principales (Nefedova et Savtchouk, 2014). Nous observons donc des effets de ségrégations et de conflits d'usage entre quartiers riches ou gated communities récents et collectifs de jardins hérités de la période soviétique, les lopins subsistant encore aujourd'hui et étant largement plébiscité par les populations les plus précaires. 
contexte met donc en lumière la structuration en cours des ségrégations sociospatiales et rend plus visibles les stratégies habitantes qui tentent d'y répondre. À Kazan, les populations urbaines créent des modes d'habiter spécifiques autour de la réappropriation des cours intérieures, de l'approvisionnement alimentaire de proximité, de la bi-résidentialité et de l'organisation de communautés afin de construire des territoires urbains en phase avec leurs aspirations. Ces modes d'habiter soulignent l'attachement des citadins russes au droit à la ville, élargi au droit à la nature, mais aussi, plus spécifiquement, au droit à la pleine terre.

\section{BIBLIOGRAPHIE}

Badyina A., Golubchikov O., 2005. Gentrification in central Moscow. A market process or a deliberate policy? Money, power and people in housing regeneration in ostozhenka. Geografiska Annaler: Series B, Human Geography, vol. 87, n² 2, p. 113-129.

Bagautdinova N., Mingazova N. M., Zamaletdinov R. I., et al., 2015. Economic, Social and Environmental Aspects of the Impact of the Universiade - 2013 on Development of Kazan City and Tatarstan Republic. Asian Social Science, vol. 11, p. 115-122.

Blanc N., Emelianoff C., 2007. Réappropriation du sol et société civile dans deux villes russes. Environnement Urbain / Urban Environment [En ligne], vol. 1. URL: http://journals.openedition.org/ eue/1049

Boukharaeva L., Marloie M., 2011. L'utilisation des sols par le jardinage urbain à grande échelle de Russie. Économie rurale. Agricultures, alimentations, territoires, n 325-326, p. 176-191.

Boukharaeva L., Marloie M., 2015. Family Urban Agriculture in Russia: Lessons and Prospects. Royaume-Uni, Springer Verlag.

Brodač M. M., Šilkin N. V., 2019. Ruralizaciâ: megatrend postindustrial'nogo obŝestva. Ènergosbereženie, $n^{\circ} 1$. URL: www.elibrary.ru/item.asp?id=36904898

Brun J., Rhein C., 1994. La ségrégation dans la ville : concepts et mesures. Paris, L'Harmattan.

Carton De Grammont S., 2013. Savoir vivre avec son temps : bref précis de cité-jardinage moscovite postsoviétique, comprenant quelques ruses symboliques de politique locale en période de libéralisation économique extrême, divers conseils sur l'art du bon voisinage avec les fantômes, ainsi qu'un menu requiem pour des efforts de bonheur. Thèse de doctorat en anthropologie. Paris, EHESS.

Carton De Grammont S., 2018. De l'Homme nouveau au Nouveau Russe : régimes de voisinage à Moscou (1924-2009). In Besse L., Cogné A, Krampl U., Sauget S., Voisiner. Mutations urbaines et construction de la cité du Moyen Âge à nos jours. Tours, Francois Rabelais (Presses universitaires), p. 243-260.

Chabrol M., Collet A., Giroud M., 2016. Gentrifications. Paris, Éditions Amsterdam.

Collinet C., Schut P.-O., 2020. L'héritage social des Jeux olympiques. Movement Sport Sciences [En ligne sur Cairn], vol. 107, n 1, p. 1-2. URL: https://www.cairn.info/revue-movement-and-sportsciences-2020-1-page-1.htm

Cottineau C., 2012. Un système intermédiaire. L'Espace géographique, vol. 41, nº 3, p. 266-284. 
Droujinin A. G., Kolossov V. A., Šuvalov V. E., 2012. Prostranstvo sovremennoj Rossii: vozmožnosti i bar'ery razvitiâ (razmyšleniâ geografov-obŝestvovedov) [L'espace de la Russie moderne : opportunités et obstacles au développement (réflexions de géographes et de spécialistes des sciences sociales)]. Moskva, Rostov-na-Donu, Vuzovskaâ kniga, 330 p.

Dussault A., 2010. Espaces contestés à Saint-Pétersbourg : quelques réactions citoyennes face à la densification urbaine. Anthropologie et Sociétés, vol. 34, n 1, p. 95-113.

Eckert D., 2012. Le monde russe. Paris, Hachette supérieur, 254 p.

Ermolaeva P., 2014. Citizen (dis)engagement during assessment of sports mega-events: the case of the 2013 Universiade in Kazan, Russia. Impact Assessment and Project Appraisal, vol. 32, $\mathrm{n}^{\circ} 1$, p. 66-71.

Failovič G. I., Igorevna K. L., 2017. Atributy sovremennogo rynka žiloj nedvižimosti [Caractéristiques du marché immobilier résidentiel moderne]. Rossijskoe predprinimatel'stvo, vol. 18, n²3, p. 3789-3798.

Faller H. M., 2002. Repossessing Kazan as a Form of Nation-building in Tatarstan, Russia. Journal of Muslim Minority Affairs, vol. 22, $\mathrm{n}^{\circ}$ 1, p. 81-90.

Golubchikov O., Badyina A., 2006. Conquering the inner-city: Urban redevelopment and gentrification in Moscow. In Tsenkova S., Nedović-Budić Z. (ed.), The Urban Mosaic of Post-Socialist Europe: Space, Institutions and Policy. Heidelberg, Physica-Verlag HD, p. 195-212.

Gorbachevskaya E. Y., 2019. Gentrification: causes and consequences for the regional housing market in Russia. IOP Conference Series: Materials Science and Engineering [En ligne], vol. 667. URL: https://iopscience.iop.org/article/10.1088/1757-899X/667/1/012027/meta

Graney K., 2007. Making Russia Multicultural Kazan at Its Millennium and Beyond. Problems of Post-Communism, vol. 54, $\mathrm{n}^{\circ}$ 6, p. 17-27.

Hilgemann F., 2007. Le Tatarstan : pays des musulmans de Russie. Paris, Éd. Autrement, 214p. Junod T., 2007. Grands événements sportifs : des impacts multiples. Finance Bien Commun, vol. 26, $\mathrm{n}^{\circ} 1$, p. 92-98.

Klyuev N. N., Nikolaevič K. N., Yakovenko L. M., 2018. "Dirty" cities in Russia: factors determining air pollution. RUDN Journal of Ecology and Life Safety, vol. 26, n 2, p. 237-250.

Kolossov V. A., 1993. La recherche urbaine en Russie : les nouveaux défis. L'Espace géographique, vol. $22, \mathrm{n}^{\circ} 4$, p. 289-298.

Kolossov V. A., 2007. La Russie : espaces, villes, réseaux. Nantes, Éd. du Temps, 319 p.

Kozovoï A., 2010. Russie : dictionnaire d'histoire et de civilisation. Paris, Ellipses.

Kutovoj S. I., 2013. Osobennosti processov urbanizacii i ruralizacii v kontekste razvitiâ gorodskogo prostranstva Rossii [Caractéristiques des processus d'urbanisation et de ruralisation dans le contexte du développement de l'espace urbain en Russie]. Kant: Èkonomika I Upravlenie [En ligne], $\mathrm{n}^{\circ}$ 1, p. 14-16. URL: www.elibrary.ru/item.asp?id=18893163

Lefebvre H., 1968. Le Droit à la ville. Paris, Edition Anthropos, 166 p.

Lovell S., 2003. Summerfolk: a history of the dacha, 1710-2000. Ithaca (NY), Cornell University Press.

Makhrova A. G., 2014. The role of organized cottage settlements in suburbanization processes in post-Soviet Russia. Regional Research of Russia, vol. 4, n 3, p. 189-197. 
Mathieu N., 2007. L'évolution des modes d'habiter : un révélateur des mutations des sociétés urbaines et rurales. In Luginbühl Y., Nouvelles urbanités, nouvelles ruralités en Europe. BruxellesBerlin, P. Lang.

Mathieu N., 2017. Les relations villes-campagnes : histoire d'une question politique et scientifique. Paris, L'Harmattan, 240 p.

Nafisa M., Derevenskaya O., Fania B., Lubov P., 2009. Restoration of Low Kaban Lake (Kazan, Russia): 25-term experience of restoration and monitoring of ecological condition [En ligne]. 13th World Lake Conference. Abstract Volume, Wuhan-China, p. 299-305. URL: https:// www.researchgate.net/profile/Olga_Derevenskaya/publication/ 264890587_Restoration_of_Low_Kaban_Lake_Kazan_Russia_25term_experience_of_restoration_and_monitoring_of_ecological_condition/links/ 5693628008aec14fa55e295a.pdf

Nefedova T. G., 2013. Desât' aktual'nyh voprosov o sel'skoj Rossii [Dix questions d'actualité sur la Russie rurale]. Moscou, NouL, $456 \mathrm{p}$.

Nefedova T. G., 2015. Rekreatsiâ gorožan kak faktor sohraneniâ i rekonstrukcii sel'skogo rasseleniâ nečernozemlâ [Loisirs des citoyens en tant que facteur de préservation et de reconstruction des régions rurales hors tchërnaziom]. Nauka. Innovatsii. Tekhnologii, $\mathrm{n}^{\circ} 2$, p. 120-139.

Nefedova T. G., Savtchouk I., 2014. Vtoroe zagorodnoe žil"'e gorožan v rossii i ukraine: èvolûciâ dač i trendy ih sovremennyh izmenenij [La maison secondaire périurbaine des citadins en Russie et en Ukraine : évolutions et tendances contemporaines]. Isvestiya RAN Seria Geografitcheskaya, $\mathrm{n}^{\circ} 4$, p. 39-49.

Nikitine A. V., Mingazova N. M., Youpina G. A., 2010. Problemy formirovania ekolovo-prirodnovo karkassa urbanizirovannykh territorii (na primere g. Kazani) [Problèmes de formations de la trame écologique et naturelle dans les espaces urbanises]. Izvestia KazGASSOU, vol. 14, $\mathrm{n}^{\circ} 2$, p. 88-96.

Ossipova A. K., Mironova A. C., Ossipova V. Y., Pritykine V. P., 2015. Prostranstvennofunkcional"'naâ organizaciâ krupnogo gradostroitel" nogo ansamblâ v novoj gorodskoj sredezapadnyj luč razvitiâ g. Kazani [Organisation spatio-fonctionnelle d'un grand ensemble dans un nouvel environnement urbain : développement occidental de Kazan]. Goroda Rossii: Problemy Stroitel' stva, Inženernogo Obespečeniâ, Blagoustrojstva I Èkologii, nº 71, p. 66-70.

Parvu S., 2016. Indefinitely Intermediate: Processes of Ruralisation in Chisinau, Moldova. Architectural Design, vol. 86, $\mathrm{n}^{\circ}$ 4, p. 28-33.

Prokofieva L., 2012. Pauvreté et inégalités en Russie. CERISCOPE Pauvreté [En ligne]. URL: http:// ceriscope.sciences-po.fr/node/390

Prokofieva L., Grichanov V., Kortchagina I., De Lapparent D., 2003. Pauvreté commune et marché à la marge en Russie. Les conditions de logement dans la Russie urbaine des années 1990. Les Annales de la Recherche Urbaine, vol. 93, $\mathrm{n}^{\circ}$ 1, p. 77-85.

Radvanyi J., 2010. La nouvelle Russie. Paris, A. Colin, 462 p.

Radvanyi J., 2018. Tatarstan, une république test du nouveau fédéralisme russe, in Chambre de commerce franco-russe [En ligne]. Annuaire de l'Observatoire franco-russe. URL: https:// hal.archives-ouvertes.fr/hal-02314424

Radvanyi J., Laruelle M., 2016. La Russie : entre peurs et défis. Paris, Armand Colin, 236 p. 
Raviot J.-R., 1993. Territoire et ethnicité au Tatarstan : une ancienne république autonome soviétique en quête d'une identité. European Journal of Sociology / Archives Européennes de Sociologie, vol. 34, n², p. 169-195.

Robert-Bœuf C., 2014. Les jardins collectifs à Kazan : une agriculture familiale entre espace productif et espace résidentiel. Pour, vol. 224, n 4, p. 101-109.

Robert-Bœuf C., 2017. Cultiver la ville en Russie. La datcha à Kazan : histoire de pratiques jardinières. Géographie et cultures, ${ }^{\circ}$ 101, p. 17-34.

Robert-Bœuf C., 2019. Les jardins collectifs : entre urbanisation de la campagne et agrarisation de la ville : mise en regard de l'Ile-de-France et de Kazan. Thèse de doctorat en géographie, Université Paris Nanterre, Nanterre.

Rochet C., 2017. Russie : la reconversion des monovilles. Constructif, vol. 48, nº 3, p. 74-77.

Solodkij A., Gorev A., 2013. System approach to elimination of traffic jams in large cities in Russia. World Applied Sciences Journal [En ligne], vol. 23, n 8. URL: http://idosi.org/wasj/ wasj23\%288\%2913/19.pdf

Stock M., 2012. « Faire avec de l'espace » : pour une approche de l'habiter par les pratiques. In Frelat-Kahn B., Lazzarotti O., Habiter : vers un nouveau concept ? Paris, A. Colin, p. 59-78.

Traven V., 2004. La datcha en Russie de 1917 à nos jours. Paris, Éd. du Sextant, 232 p.

Vendina O., 2007. Système urbain : enjeu de la modernisation. In Kolossov V. A., La Russie : espaces, villes, réseaux. Nantes, Éd. du Temps.

Zakirova T. R. 2015. Razvitie gradostroitel'noj struktury goroda Kazani s serediny 1930-h do serediny 1950-h godov [Développement des structures urbaines de la ville de Kazan du milieu des années 1930 au milieu des années 1950]. Izvestiya Kazanskovo gossoudarstvennovo arkhitektournostroitel'novo Ouniversiteta [En ligne], vol. 31, n 1, p. 6-10. URL: https://cyberleninka.ru/article/n/ razvitie-gradostroitelnoy-struktury-goroda-kazani-s-serediny-1930-h-do-serediny-1950-h-godov

Žel'kovič Z. A., 2016. Analiz obespečennosti gorodov-millionnikov Rossii transportnoj infrastrukturoj [Analyse des infrastructures de transport dans les villes de plus d'un million d'habitants]. Vestnik Moskovskogo universiteta imeni S.Û. Vitte, Seriâ 1: Èkonomika i upravlenie [En ligne], vol. 17, n². URL: https://cyberleninka.ru/article/n/analiz-obespechennosti-gorodovmillionnikov-rossii-transportnoy-infrastrukturoy

\section{NOTES}

1. Toutes les statistiques officielles russes ont été consultées sur : https://rosstat.gov.ru/statistic. Toutes les statistiques officielles sur le Tatarstan et sur Kazan ont été consultées sur: https:// tatstat.gks.ru/ofstatistics

2. Crise qui débute avec la chute de l'URSS en 1991 et qui est aggravée en 1998 par la dévaluation brutale du rouble et un défaut sur la dette russe.

3. Voir les statistiques officielles disponibles sur https://rosstat.gov.ru/accounts et l'article suivant : https://lenta.ru/articles/2019/07/31/hardmsk/

4. Terme russe désignant les propriétaires de datcha qui cultivent un potager. Dačniki est le pluriel de dačnik. Il sera considéré ici comme un synonyme de jardinier. Les termes en cyrillique ont été transcrits selon le système de translittération ISO 9, qui fait référence dans les publications scientifiques françaises.

5. Les chiffres ne sont cependant pas disponibles. 
6. Par respect de l'anonymat, tous les prénoms et les noms ont été changés.

7. Subdivision administrative que l'on pourrait traduire par région en français ou par quartier (ou arrondissement) quand on l'utilise à l'échelon de la ville (Kozovoï, 2010).

8. Dans le collectif de jardins de Borovoe Matiochino, des dačniki ont subi des pressions pour vendre leur parcelle à des voisins plus aisés qui souhaitaient agrandir leurs parcelles, cela a donné lieu à un procès en 2013.

9. Ce phénomène peut également être le signe d'un processus de blanchiment d'argent dans le secteur de l'immobilier, mais nos enquêtes ne permettent pas de l'affirmer.

\section{RÉSUMÉS}

La Russie a connu un processus d'urbanisation marqué par la crise économique des années 1990 et, plus récemment, par la métropolisation des grandes villes. Kazan, capitale du Tatarstan, offre un bon exemple de ces nouvelles métropoles en essor, car depuis 2005 elle a connu d'importantes transformations urbaines, devenant le symbole des Tatars de Russie. Son évolution et les réaménagements successifs ont créé de fortes inégalités socio-spatiales et environnementales, visibles notamment à travers l'étude des collectifs de jardins. Pour y répondre, les citadins et jardiniers mettent en place des stratégies individuelles et collectives de manière à construire des modes d'habiter correspondant mieux à leurs aspirations.

Russia has experienced a recent urbanization process affected by the economic crisis of the 1990s and, more recently, by the metropolization of large cities. Kazan, capital of Tatarstan, offers a good example of these new growing metropolises. Indeed, since 2005, Kazan has undergone major urban transformation, becoming Russian Tatars' symbol. Nevertheless, evolution and successive redevelopments created strong socio-spatial and environmental inequalities, visible in particular through allotment gardens' analyzes. Thus, urban populations and gardeners are implementing individual and collective strategies in order to build better ways of inhabiting matching their aspirations.

\section{INDEX}

Mots-clés : métropolisation, inégalité, mode d'habiter, datchas, Kazan

Keywords : metropolization, inequality, way of inhabiting, dachas, Kazan

\section{AUTEUR}

\section{CAMILLE ROBERT-BOEUF}

Camille Robert-Boeuf, Camille Robert-Boeuf, est postdoctorante au CNRS - UMR Ladyss et chercheuse associée au laboratoire KOuRT de l'Université Fédérale de Kazan. Elle a récemment publié :

- Robert-Boeuf C., 2017. Cultiver la ville en Russie. La datcha à Kazan : histoire de pratiques jardinières. Géographie et Cultures, $n^{\circ}$ 101, p. 17-34. 
- Robert-Boeuf C., 2017. Les jardins collectifs en Ile-de-France et à Kazan. Des espaces hybrides pour réinterroger les relations villes-campagnes. In Berger M. et Chaléard J.-L. (dir), Villes et campagnes en relations : regards croisés Nords-Suds. Paris, Ed. Karthala, p. 200-212.

- Robert-Boeuf C., 2017. Les jardins collectifs constitutifs de réseaux alternatifs au service des urbains. Étude de cas de l'Ile-de-France et de Kazan (Russie). In Gauvard C. (dir.), Appartenances et pratiques des réseaux. Éditions électroniques du CTHS, p. 205-217. 